\title{
Medical and gastroenterological education during the COVID-19 outbreak
}

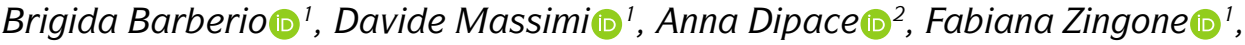 \\ Fabio Farinati $\mathbb{1}^{\prime}{ }^{\prime}$ and Edoardo V. Savarino (iD ${ }^{1 凶}$
}

The outbreak of coronavirus disease 2019 has undeniably affected modalities of medical and gastroenterology training in all endemic areas. Indeed, the adoption of various distance-learning techniques has been mandatory to facilitate education and enhance skills such as problem-solving, self-directed learning, open communication and also holistic non-cognitive attributes such as adaptability and collaboration.

The coronavirus disease 2019 (COVID-19) outbreak, caused by infection from the severe acute respiratory syndrome coronavirus 2 (SARS-CoV-2), is an ongoing pandemic that, in just a few months, has dramatically changed many aspects of our lifestyles, including education. It was immediately clear to the entire scientific community that it was necessary to reorient study and research priorities towards the COVID-19 outbreak, so as to make vital information and knowledge available. For the longer term, however, the future of medical education at all levels is unclear. Indeed, the beginning of this health crisis has interrupted and distorted the learning and training routines in hospitals and universities, leaving students and teachers with a lot of uncertainty ${ }^{1,2}$.

As of early April 2020, with SARS-CoV-2 spreading around the world, the education system in many endemic areas faced a mandatory total closure of in-person activities to adapt to social distancing measures. Worldwide, more than one billion students ended their participation in teaching or curricular activities in schools and universities, and all activities were rescheduled (UNESCO COVID-19 Educational Disruption and Response). In particular, all universities suspended their traditional in-person teaching and offered students online lessons to guarantee their right to study. They also engaged in the arduous task of conducting examinations, organizing graduation sessions, preparing internships and virtual laboratories, planning activities guidance and offering support to students with special educational needs. These tasks were often possible through methods of distance learning and, therefore, conducted strictly online. Digital online platforms were also used to maintain in-person lessons, in some cases also trying to maintain the possibility of evaluating proficiency with oral examinations via webcam.
In parallel, specific COVID-19-oriented training has been implemented through the design of webinars and the publication of various educational resources via the large number of digital platforms available (see Related links). Further, online problem-based learning techniques have been implemented to enhance problem-solving and critical thinking, improve clinical skills, encourage self-directed learning skills, compensate for lack of rotations in outpatient ambulatories and complete the curricula for some final year medical students or postgraduates who were in the process of appearing for their final assessments ${ }^{3}$.

According to the Accreditation Council for Graduate Medical Education (ACGME) in the USA, which is an independent, physician-led organization that establishes and monitors the professional educational standards essential in preparing physicians, one in seven active physicians in the USA is a resident or fellow ${ }^{4}$. Thus, trainee physicians in the university hospitals certainly represent a vital figure in both this crisis and more broadly in health-care dynamics, as they have been widely involved in the frontline.

The COVID-19 outbreak requires a reassessment of the current medical specialist training course. The elimination and reduction of most elective procedures, such as surgical interventions apart from oncological and urgent cases, and outpatient visits, has inevitably jeopardized the development of specialist skills, at least temporarily. Moreover, with the establishment of dedicated COVID-19 departments, postgraduates and fellows from medical areas such as gastroenterology have been re-distributed. Of course, gastroenterology fellows already in charge of the management of acute patients and emergencies referred to their hospitals (for example, those with gastrointestinal bleeding, acute pancreatitis, inflammatory bowel disease flares and 


\section{Box 1 | Learning measures to adopt during the COVID-19 pandemic}

Wards and consultations

- Guarantee involvement and collaboration in clinical activities in coronavirus disease 2019 (COVID-19)

- Maintain non-elective gastrointestinal activities but limit trainee involvement and preserve protective measures

- Implement telemedicine for inpatient and outpatient consultations

- Weekly rescheduling of activities to minimize team handoffs

Endoscopic training

- Maintain non-elective endoscopy activities, but limit trainee involvement and preserve protective measures

- Implement simulation-based training opportunities (for example, virtual reality simulator models)

\section{Distance learning}

- Local web-based lectures (for example, grand rounds, online graduation sessions, journal clubs, fellow core lectures and clinical cases through problem-based learning techniques)

- Video-based education (for example, recorded endoscopy cases) with interaction

- National and international webinars or lectures, primarily via gastrointestinal societies
Finally, in the case of specialist training in gastroenterology, it is necessary to deal with an important topic: endoscopic training. Different publications in the past few months have suggested the potential risk of infections associated with the execution of endoscopic procedures $^{6,7}$. All these issues resulted in the abrupt cessation of non-urgent elective endoscopic procedures, triggering a decline in the number of weekly procedures performed and an immediate repercussion on endoscopic training. Hospital and training schools have been forced to suspend and reschedule all trainings to a later, unspecified date. Only a few gastroenterology units continued to carry out outpatient procedures, but often excluding fellows. For instance, in North America, a substantial number of endoscopy units eliminated their training during this pandemic ${ }^{8}$. A critical yet constructive look at the emerging problem of endoscopic training in the COVID-19 era suggests two solutions. The first, currently implemented, is the enhancement of virtual training, with webinars held by specialist scientific societies in an attempt to strengthen the theoretical background to endoscopy. For example, the Italian Society of Gastroenterology and Endoscopy (SIGE) organized a periodic webinar called the 'School of Advanced Endoscopy - Meet the Experts around the world', in which experts in endoscopy provided special lessons with question and answer sessions to teach specific topics in endoscopy. SIGE also set up an e-learning platform to be used to deliver lectures and tutorials remotely via phones, tablets and laptops. Thus, faculty residents and medical students can log in at designated times for discussions, which can be facilitated in real time via teleconferencing applications. In addition to lectures, teleconferencing can also be used to demonstrate medical procedures and endoscopic techniques.

Similarly, in a gastroenterology unit in Philadelphia, USA, they provided fellows of the American Society for Gastrointestinal Endoscopy with video sessions that reviewed common technical aspects of endoscopy ${ }^{5}$. For third-year fellows still looking to meet requirements for less commonly performed procedures, such as percutaneous endoscopic gastrostomies, they reached out to their surgical colleagues with the hope of gaining access to a greater volume of these procedures when able to participate in procedures again. Equally, the American Gastroenterological Association (AGA) has offered an educational web destination for fellows (see Related links).

The second measure, which at the moment is a proposal rather than a reality, is to try to keep active this crucial practical application through the enhancement and rediscovery of simulation-based training. The literature provides a large amount of data supporting the effectiveness of simulated training in enhancing the endoscopy training path, often with excellent results ${ }^{9,10}$. In particular, virtual reality simulator technology has been implemented in the past few years, providing the possibility of performing endoscopies remotely. Based on this model, a comprehensive curriculum that teaches technical, cognitive and integrative skills (for example, communication) in colonoscopy has been developed to ensure transfer of skills to the clinical setting. 
The COVID-19 outbreak is undeniably affecting medical training, but contingencies should be undertaken to reduce disruptions (BOX 1). Technology and distance learning can be implemented to facilitate medical instruction as well as courses enhancing skills such as problem-solving, critical thinking, self-directed learning and open communication. Moreover, the involvement of medical students, residents and fellows in pandemic efforts can be beneficial for the development of their clinical skills and also holistic non-cognitive attributes, such as adaptability and collaboration.

1. Ferrel, M. N. \& Ryan, J. The impact of COVID-19 on medical education. Cureus 12, e7492 (2020)

2. Ahmed, H., Allaf, M. \& Elghazaly, H. COVID-19 and medical education. Lancet Infect. Dis. https://doi.org/10.1016 S1473-3099(20)30226-7 (2020).

3. Wilder, S. Impact of problem-based learning on academic achievement in high school: a systematic review. Educ. Rev. 67, 414-435 (2015)

4. Shah, R. et al. COVID-19 pandemic through the lens of a gastroenterology fellow: looking for the silver lining. Gastrointest. Endosc. https://doi.org/10.1016/j.gie.2020.03.3852 (2020).

5. Palchaudhuri, S. et al. Adapting a Gl fellowship to a pandemic: novel approaches to accommodating a novel virus. Dig. Dis. Sci. 65, 1562-1565 (2020).

6. Gu, J., Han, B. \& Wang, J. COVID-19: gastrointestinal manifestations and potential fecal-oral transmission. Gastroenterology 158 , 1518-1519 (2020).

7. Xiao, F. et al. Evidence for gastrointestinal infection of SARS-CoV-2. Gastroenterology 158, 1831-1833 (2020).
8. Forbes, N. et al. Changes in gastroenterology and endoscopy practices in response to the covid-19 pandemic: results from a North American survey. Gastroenterology https://doi.org/10.1053/ j.gastro.2020.04.071 (2020).

9. Siau, K. et al. Impact of a simulation-based induction programme in gastroscopy on trainee outcomes and learning curves. World J. Gastrointest. Endosc. 12, 98-110 (2020).

10. Grover, S. C. et al. Impact of a simulation training curriculum on technical and nontechnical skills in colonoscopy: a randomized trial. Gastrointest. Endosc. 82, 1072-1079 (2015).

\section{Author contributions}

B.B. and E.V.S. conceived the article. B.B., A.D., D.M., F.Z., E.V.S. drafted the manuscript. All authors commented on drafts of the paper and approved the final version.

Competing interests

The authors declare no competing interests.

\section{RELATED LINKS}

American Gastroenterology Association resources for COVID-19:

https://www.gastro.org/practice-guidance/practice-updates/covid-19

European Association for the Study of Liver Disease, COVID-19 and the

liver: https://easl.eu/covid-19-and-the-liver/

European Society of Eosinophilic Esophagtis recommendations:

https://eureos.online/home-eureos.html

European Society of Gastrointestinal Endoscopy webinars: www.esge.com

Società Italiana di Gastroenterologia ed Endoscopia Digestiva,

School of advanced endoscopy - meet the experts around the world:

https://www.sigeitalia.it/

United European Gastroenterology resources for COVID-19:

https://ueg.eu/p/153

UNESCO COVID-19 Educational Disruption and Response:

https://en.unesco.org/covid19/educationresponse 